\title{
In Silico Approach Towards the Prediction of Drug-likeness, in Vitro Microbial Investigation and Formation of Dihydropyrrolone Conjugates
}

\author{
Keyur Pandya a,* (D), Bhavesh Daveb ${ }^{b}$ Rajesh Patelc, Piyush Desaia \\ a Department of Chemistry, Arts, Science, and Commerce College, Veer Narmad South Gujrat University, Surat, Gujrat, \\ India \\ b Department of Chemistry, M.B. Patel Science College, Sardar Patel University, Anand, Gujarat, India \\ c Department of Organic Chemistry, Shri A.N. Patel P.G. Institute of Research and Sciences, Anand, Gujarat, India
}

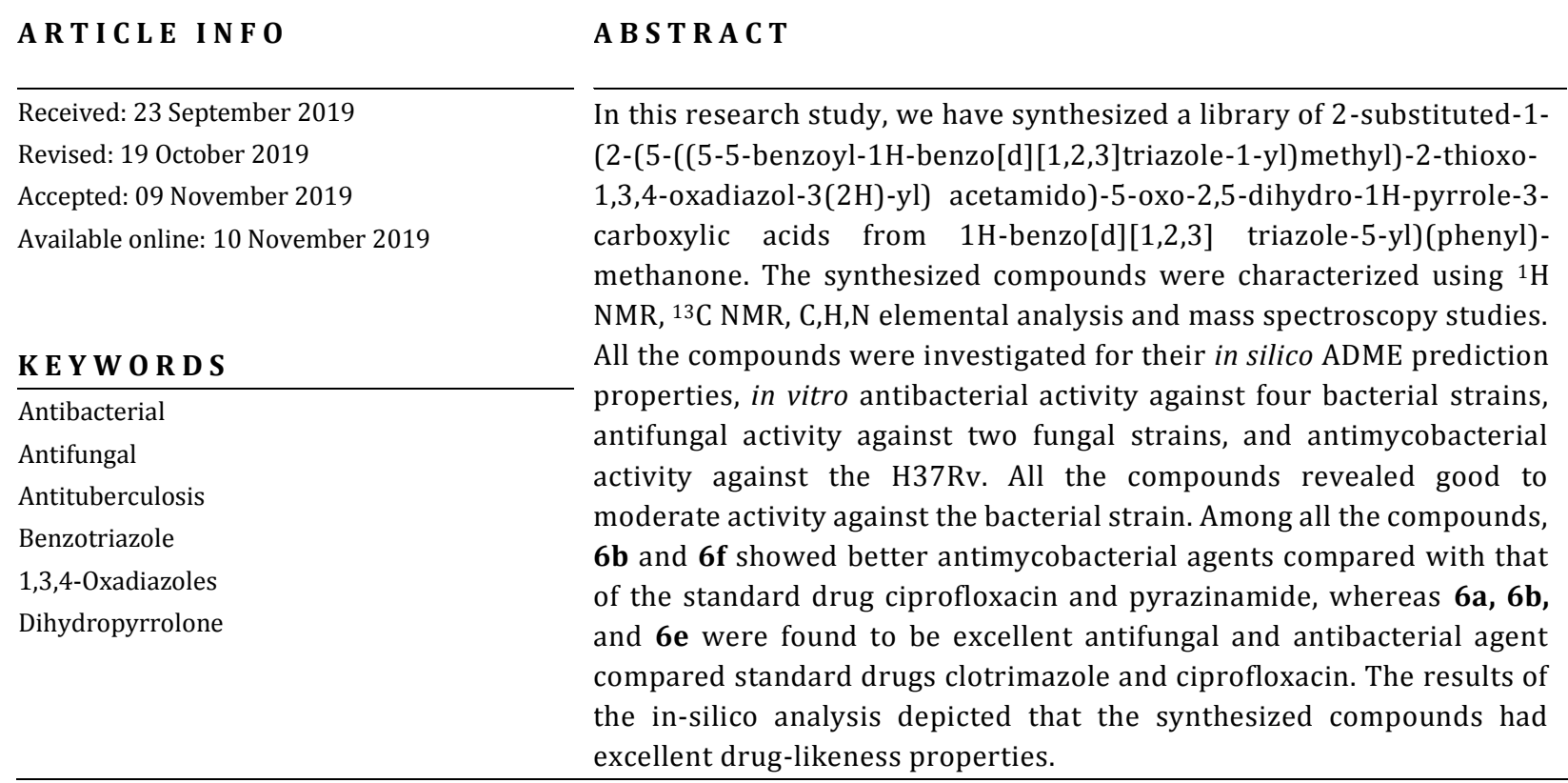

\section{G R A P H I C A L A B S T RA C T}

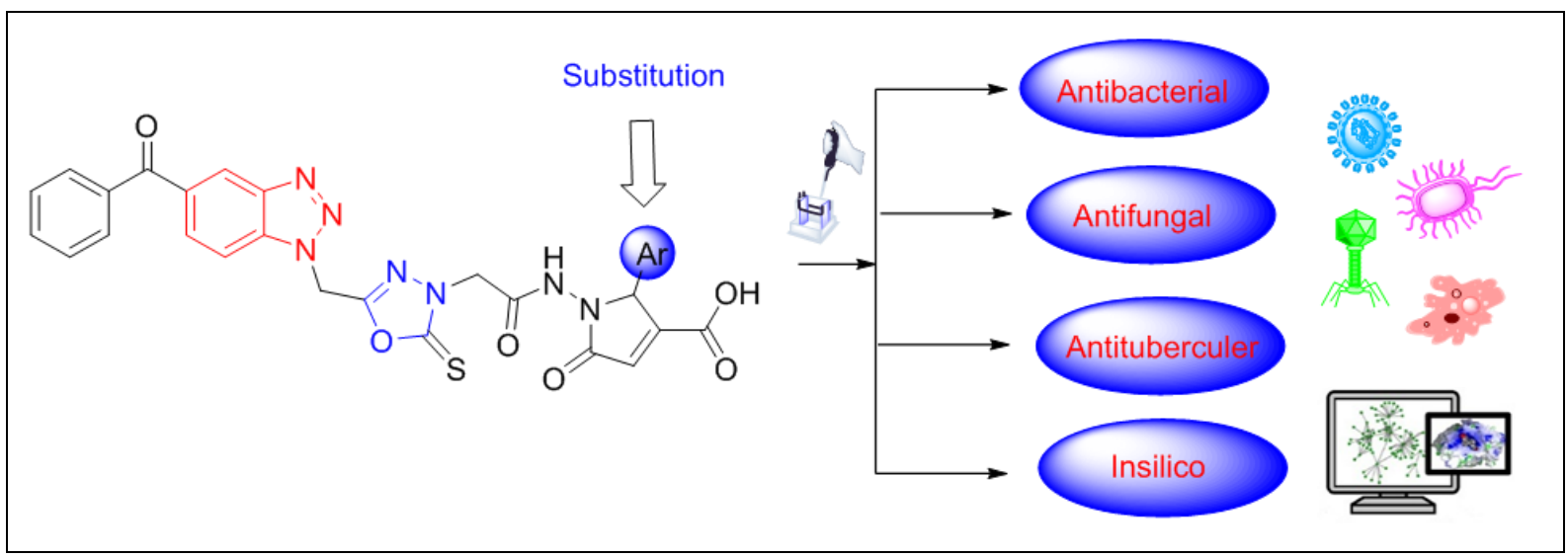

* Corresponding author's E-mail address: scientistatwork@outlook.com 


\section{Introduction}

Heterocyclic molecules are cyclic molecules, containing one or more heteroatoms besides the carbon atom. The most common heteroatoms are nitrogen, oxygen, and sulphur. The heterocyclic compounds containing three to six carbons in the ring are numerous; however, those containing five or six atoms are very important in the pharmaceutical world. Pyrroles demonstrate such flexibility (Figure 1) with valuable substructure in a collection of pharmaceuticals, involving products potent against HIV [1,2], influenza [3], cytomegalovirus [4], anticancer agents $[5,6]$ and compounds effective against microbiological infections [7-9] such as bacterial and fungal. In addition, pyrroles are known as building blocks in the synthesis of alkaloids [10-13] and intermediates including, 2,2'-bipyrroles, pyrroles, and pigments [14-19]. Structural alteration of the pyrrole ring to create more bioactive molecules has attracted agreat deal of attention from many researchers. Over the years much effort has been directed towards developing new strategies for 2-arylpyrrole and oligopyrrole synthesis [20-27].

Continuing interest in the development of the synthetic methodology of dihydropyrrole synthesis provides some impetus to initiate a project for developing a new and more expedient route for the pyrrole rings syhthesis. Dihydropyrroles may be employed as important synthetic intermediates for synthesis of pyrroles. Substituted dihydropyrroles are a vital class of five membered nitrogen-containing heterocycles as they are present in numerous bioactive compounds and pharmaceuticals, such as sibiromycin [28], anthramycin [29], serotonin reuptake inhibitor [30], and thienamycin [31]. Furthermore, dihydropyrroles can be used as versatile synthetic intermediates for the synthesis of natural products [32]. Therefore, considerable efforts have been devoted to the synthesis of these heterocyclic motifs, establishing numerous synthetic methods. Among these techniques, the commonly used approaches are cyclization reactions. Related dihydropyrrol-2-one (DHP) analogues, which bear a nitrogen atom in place of the oxygen in the heterocyclic ring, are more hydrolytically stable under physiological conditions [33]. DHP is a common moiety in several classes of biologically active molecules such as pulchellalactam (1a-b, Figure 2), jatropham, and rolipram [34-36]. Novel dihydropyrrolone derivatives have been developed in our laboratory (Figure 3) and their bioactivities as well as in silico propertes are discussed in this article.

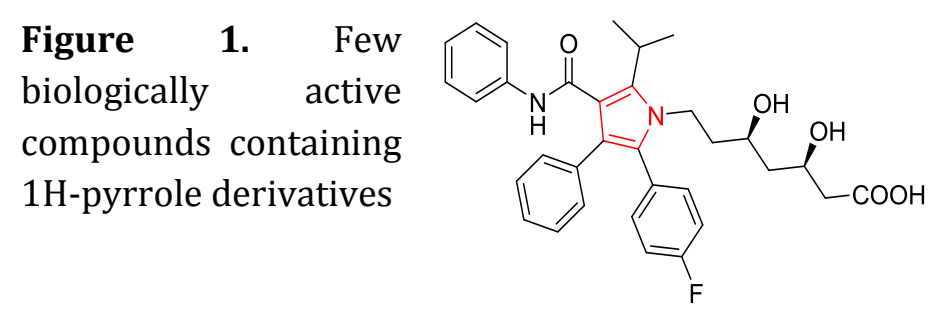

Atorvastatin

use to control production of cholesterol the body

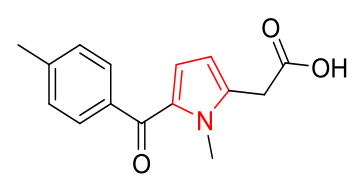

Tolmetin nonsteroidal anti-inflammatory

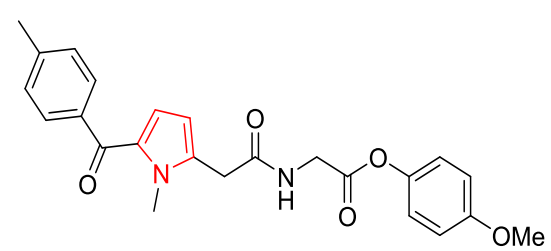

Amtolmetin

use to treat arthritis

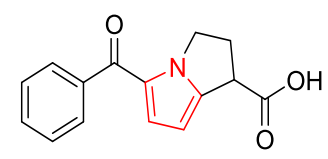

Ketorolac

use to treat eye pain and itchiness 
Figure 2. Biologically active pyrrole-2-ones

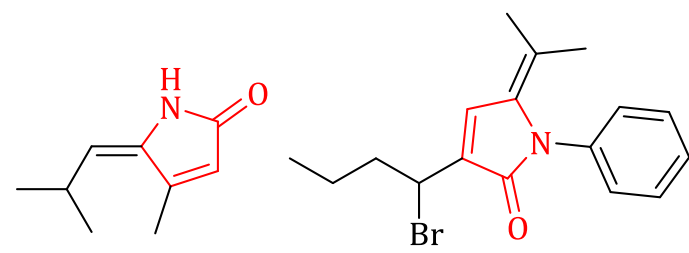

$1 \mathrm{a}$

Figure 3. Design strategy for target compounds

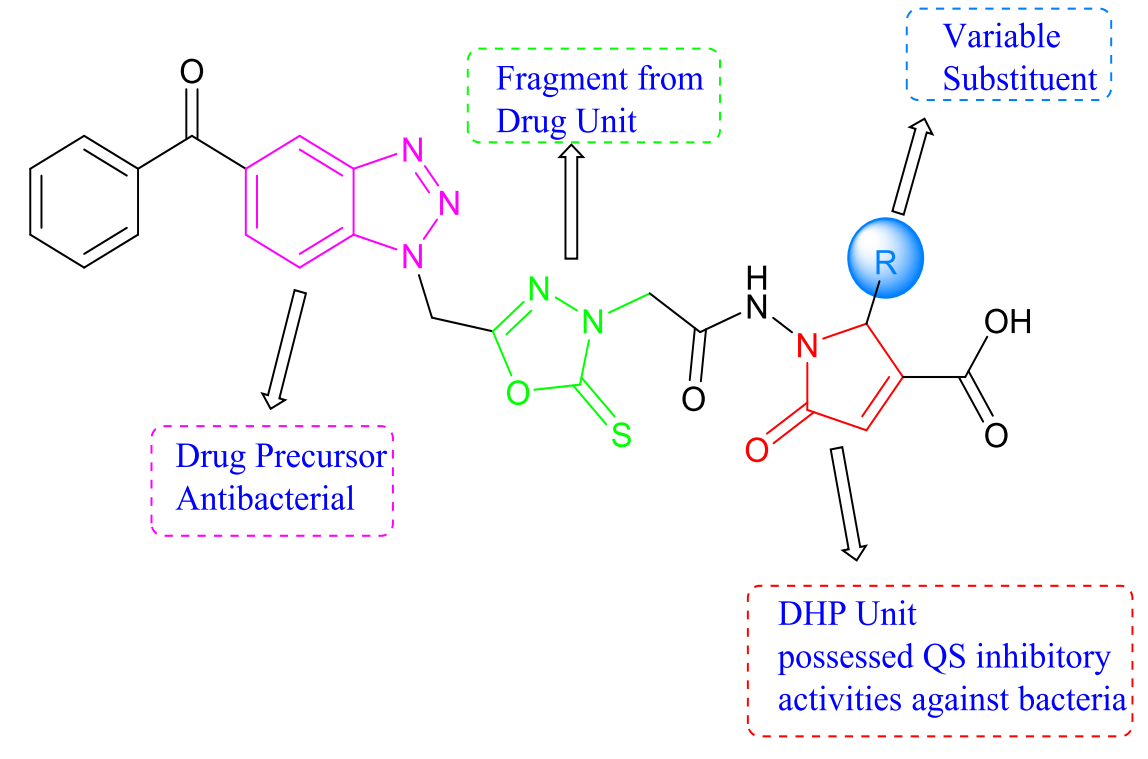

\section{Experimental}

\section{Materials and methods}

All the reactants were purchased from Sigma Aldrich, and used without further purification. All solvents were used without further drying or purification and were of ACS grade purchased from local suppliers. TLC plates (silica gel) were purchased from Sigma-Aldrich. Melting points were determined in open capillary tubes on a Stuart SMP 10 melting point apparatus. Nuclear magnetic spectroscopy (NMR) spectra were produced using the Varian $300 \mathrm{MHz}$ spectrophotometer. The instrument was maintained at $25^{\circ} \mathrm{C}$ operating at $300 \mathrm{MHz}$ for ${ }^{1} \mathrm{H}$ $\mathrm{NMR}$, and $75 \mathrm{MHz}$ for ${ }^{13} \mathrm{C}$ NMR. The deuterated solvent (DMSO- $\mathrm{d}_{6}$ ) used for each respective spectrum was referenced to the appropriate literature peak shift.

General procedure for synthesis of 2-(5benzoyl-1H-benzo[d][1,2,3]triazol-1yl)acetohydrazide. 2
To the solution of $(1 \mathrm{H}-$ benzo[d][1,2,3]triazol-5

yl)(phenyl)methanone (22.4 mmol, $5 \mathrm{~g}$ ) in absolute EtOH $\left(60 \mathrm{~cm}^{3}\right)$, methyl chloroacetate (22.4 mmol, $1.96 \mathrm{~cm}^{3}$ ), hydrazine monohydrate (22.4 mmol, $\left.1.09 \mathrm{~cm}^{3}\right)$ and anhydrous $\mathrm{K}_{2} \mathrm{CO}_{3}(26.9 \mathrm{mmol}, 3.7 \mathrm{~g})$ were added and the reaction mixture was heated under reflux for $16 \mathrm{~h}$. Progress of the reaction was noticed by TLC technique. After completion of the reaction, the potassium salt was filtered off and the excess of ethanol was removed. The residue solidified in cold water, dried and, recrystallized by EtOH to collect the final product 2 in good yields (5.3 g, 80.13\%) mp 145-147 $\circ \mathrm{C},{ }^{1} \mathrm{H}$ NMR (300 MHz, DMSO-d6) $\delta 9.14(\mathrm{t}, J=4.3 \mathrm{~Hz}, 1 \mathrm{H}), 8.33(\mathrm{~d}, J=1.6 \mathrm{~Hz}, 1 \mathrm{H})$, 7.90 (dd, $J=7.5,1.5 \mathrm{~Hz}, 1 \mathrm{H}), 7.84-7.74(\mathrm{~m}$, $3 \mathrm{H}), 7.63-7.43(\mathrm{~m}, 3 \mathrm{H}), 4.90(\mathrm{~s}, 2 \mathrm{H}), 4.19(\mathrm{~d}$, $J=4.3 \mathrm{~Hz}, 2 \mathrm{H})$.

General procedure for the synthesis of phenyl(1((5-thioxo-4,5-dihydro-1,3,4-oxadiazol-2- 
yl)methyl)-1H-benzo[d][1,2,3]triazol-5yl)methanone. 3

A Mixture of compound 2 (10.2 mmol, $3 \mathrm{~g}$ ) was added to $\mathrm{MeOH}\left(100 \mathrm{~cm}^{3}\right)$, potassium hydroxide (5.34 mmol, $0.3 \mathrm{~g}$ ) and heated with $\mathrm{CSCl}_{2}\left(5.21 \mathrm{mmol}, 0.38 \mathrm{~cm}^{3}\right)$ and refluxed for about $12 \mathrm{~h}$ at $65^{\circ} \mathrm{C}$. Progress of the reaction was monitored using the TLC technique. After completion of the reaction, the separated solid was filtered, dried in vacuum, and purified over a column of silica gel, eluted with $\mathrm{C}_{6} \mathrm{H}_{6}: \mathrm{CHCl}_{3}$ $(2: 8 \mathrm{v} / \mathrm{v})$ mixture to give a final product 3 with yield of $(2.81 \mathrm{~g}, 81.92 \%) . \mathrm{mp} 177-179{ }^{\circ} \mathrm{C},{ }^{1} \mathrm{H}$ NMR (300 MHz, DMSO-d $d_{6} \delta 12.39$ (s, 1H), 8.31 (d, $J=1.6 \mathrm{~Hz}, 1 \mathrm{H}$ ), 7.91 (dd, $J=7.5,1.5 \mathrm{~Hz}, 1 \mathrm{H}$ ), 7.85-7.77 (m, 3H), 7.63-7.56 (m, 1H), 7.54-7.48 $(\mathrm{m}, 2 \mathrm{H}), 4.74(\mathrm{~s}, 2 \mathrm{H})$.

General procedure for the synthesis of 2-(5-(c5benz oyl-1H-benzo[d][1,2,3]triazol-1yl)methyl)-2-thioxo-1,3,4-oxadiazol-3(2H)yl)acetohydrazide. 4

To the solution of reactant $3(7.11 \mathrm{mmol}, 2.4$ g) in absolute EtOH $\left(60 \mathrm{~cm}^{3}\right)$, methyl chloroacetate $\left(7.11 \mathrm{mmol}, 0.4 \mathrm{~cm}^{3}\right)$, hydrazine monohydrate $\left(7.11 \mathrm{mmol}, 0.65 \mathrm{~cm}^{3}\right)$ and anhydrous $\mathrm{K}_{2} \mathrm{CO}_{3}(8.54 \mathrm{mmol}, 1.2 \mathrm{~g})$ were added and the reaction mixture was heated under reflux for $16 \mathrm{~h}$. Progress of the reaction was noticed by TLC technique. After completing the reaction, the potassium salt was filtered off and the excess of ethanol was removed. The residue solidified in cold water, dried, and recrystallized using EtOH to collect the final product 4 in good yields (2.4 g, 82.39\%). mp 186-188 ${ }^{\circ} \mathrm{C}$, ${ }^{1} \mathrm{H}$ NMR $\left(300 \mathrm{MHz}, \mathrm{DMSO}-d_{6}\right) \delta 9.23(\mathrm{t}, J=5.1 \mathrm{~Hz}, 1 \mathrm{H})$, 8.22 (d, $J=1.6 \mathrm{~Hz}, 1 \mathrm{H}), 7.92$ (dd, $J=7.5,1.5 \mathrm{~Hz}$, 1H), $7.83-7.75(\mathrm{~m}, 3 \mathrm{H}), 7.63-7.54(\mathrm{~m}, 1 \mathrm{H})$, $7.54-7.46(\mathrm{~m}, 2 \mathrm{H}), 4.82(\mathrm{~s}, 2 \mathrm{H}), 4.32(\mathrm{~s}, 2 \mathrm{H})$, $4.23-4.10(\mathrm{~m}, 2 \mathrm{H})$.

General procedure for the synthesis of (E)- $N^{\prime}$ (substituted methylene)-2-(5-((5-benzoyl-1Hbenzo[d][1,2,3]triazol-1-yl)methyl)-2-thioxo1,3,4-oxadiazol-3(2H)-yl)acetohydrazid. 5a-h
A mixture of compound $4(0.01 \mathrm{~mol})$ and benzaldehyde $(0.01 \mathrm{~mol})$ was made in absolute ethanol $(100 \mathrm{~mL})$ and was refluxed for about 4$8 \mathrm{~h}$ at $79{ }^{\circ} \mathrm{C}$ with a catalytic amount of glacial acetic acid (1-3 drops) on a water bath. The reaction was monitored using the TLC. The mixture was evaporated under the reduced pressure to give a residue. The residue was dissolved in DCM, and the organic layer were dried over $\mathrm{Na}_{2} \mathrm{SO}_{4}$, filtered, and concentrated. The residue was purified using the silica gel chromatography $\quad(\mathrm{PE} / \mathrm{EtOAc}=1: 1) \quad$ and recrystallized from $\mathrm{PE} / \mathrm{EtOAc}$ to afford product 5a. Similarly, 5b-h was prepared using the same procedure and different aromatic aldehydes (as listed in Scheme 1).

General procedure for the synthesis of 2substituted-1-(2-(5-)([5-benzoyl-1H-benzo[d] [1,2,3]triazol-1-yl)methyl)-2-thioxo-1,3,4oxadiazol-3(2H)-yl)acetamido)-5-oxo-2,5dihydro-1H-pyrrole-3-carboxylic acid. 6a-h

A mixture of reactant $5 \mathbf{5 a}(0.135 \mathrm{~mol})$ and maleic anhydride $(0.15 \mathrm{~mol})$ was dissolved in anhydrous toluene $(150 \mathrm{~mL})$ and stirred under nitrogen during $24 \mathrm{~h}$ at $35^{\circ} \mathrm{C}$. The mixture was cooled at room temperature and the solid was separated by filtration, and purified by extraction with ethyl acetate after addition of sodium carbonate. The aqueous layer was treated with phosphoric acid. The white solid product separated by filtration with ice-cold water, then dried and recrystallized in $45 \%$ ethanol to obtain white crystals of compound 6a. Similarly, other compounds $\mathbf{6 b}-\mathbf{h}$ were prepared using the same procedure with different reactants $\mathbf{5 b} \mathbf{b} \mathbf{h}$.

Reagents and conditions (a) $\mathrm{EtOH}$, $\mathrm{ClCH}_{2} \mathrm{COOCH}_{3}, \mathrm{NH}_{2} \mathrm{NH}_{2} . \mathrm{H}_{2} \mathrm{O}$, Anhy. $\mathrm{K}_{2} \mathrm{CO}_{3}$, reflux, 16 hrs. (b) $\mathrm{MeOH}, \mathrm{KOH}, \mathrm{CSCl}_{2}$, reflux, 12 hrs. (c) EtOH, $\mathrm{ClCH}_{2} \mathrm{COOCH}_{3}, \mathrm{NH}_{2} \mathrm{NH}_{2} . \mathrm{H}_{2} \mathrm{O}$, Anhy. $\mathrm{K}_{2} \mathrm{CO}_{3}$, reflux, 16 hrs. (d) Aromatic aldehyde, EtOH, glacial acetic acid, reflux, 4-5 hrs (e) maleic anhydride, anhydrous toluene, under $\mathrm{N}_{2}, 24 \mathrm{hr}, 35^{\circ} \mathrm{C}$, 54-68\%. 
Scheme 1. General synthetic route of the title compounds
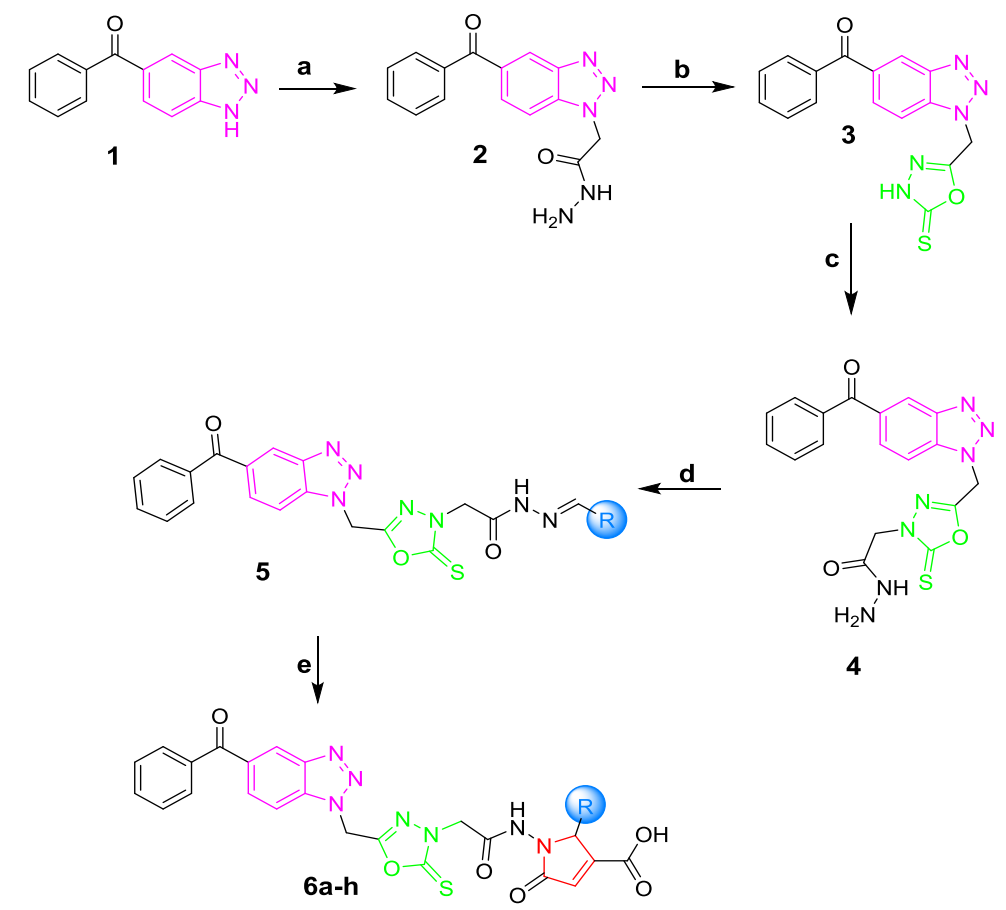

4

\begin{tabular}{cc} 
& $\mathrm{R}-$ \\
$\mathrm{a}$ & $\mathrm{C}_{6} \mathrm{H}_{5^{-}}$ \\
$\mathrm{b}$ & $\mathrm{p}-\mathrm{Cl} \mathrm{C}_{6} \mathrm{H}_{4^{-}}$ \\
$\mathrm{c}$ & $\mathrm{p}-\mathrm{CH}_{3} \mathrm{C}_{6} \mathrm{H}_{4}-$ \\
$\mathrm{d}$ & $\mathrm{p}-\mathrm{OCH}_{3} \mathrm{C}_{6} \mathrm{H}_{4-}^{-}$ \\
$\mathrm{e}$ & 2-thienyl \\
$\mathrm{f}$ & $\mathrm{o}-\mathrm{Cl} \mathrm{C}_{6} \mathrm{H}_{5^{-}}$ \\
$\mathrm{g}$ & $\mathrm{p}-\mathrm{CH}_{2} \mathrm{CH}_{3} \mathrm{C}_{6} \mathrm{H}_{5^{-}}$ \\
$\mathrm{h}$ & $\mathrm{p}-\mathrm{OCH}_{2} \mathrm{CH}_{3} \mathrm{C}_{6} \mathrm{H}_{5}$ \\
\hline
\end{tabular}

Spectral and physical data for compounds

1-(2-(5-((5-benzoyl-1H-benzo[d][1,2,3]triazol1-yl)methyl)-2-thioxo-1,3,4-oxadiazol-3(2H)yl)acetamido)-5-oxo-2-phenyl-2,5-dihydro- $1 \mathrm{H}$ pyrrole-3-carboxylic acid (6a)

Yield: $61 \%$; white solid, m.p. $174-175^{\circ} \mathrm{C}$; ${ }^{1} \mathrm{H}$ NMR (300 MHz, DMSO- $\left.d_{6}\right) \delta(\mathrm{ppm}) 10.30$ (s, $1 \mathrm{H}), 8.44-8.40(\mathrm{~m}, 1 \mathrm{H}), 8.12-8.06(\mathrm{~m}, 1 \mathrm{H})$, $7.89(\mathrm{dd}, J=10.2,2.3 \mathrm{~Hz}, 1 \mathrm{H}), 7.84-7.78(\mathrm{~m}$, $2 \mathrm{H}), 7.63-7.56(\mathrm{~m}, 1 \mathrm{H}), 7.54-7.47(\mathrm{~m}, 2 \mathrm{H})$, $7.43(\mathrm{~s}, 5 \mathrm{H}), 6.69$ (d, $J=1.7 \mathrm{~Hz}, 1 \mathrm{H}), 5.56$ (d, $J$ $=1.7 \mathrm{~Hz}, 1 \mathrm{H}), 4.69(\mathrm{~d}, J=17.6 \mathrm{~Hz}, 1 \mathrm{H}), 4.52-$ $4.40(\mathrm{~m}, 2 \mathrm{H}), 4.23(\mathrm{~d}, J=13.6 \mathrm{~Hz}, 1 \mathrm{H}) .{ }^{13} \mathrm{C} \mathrm{NMR}$ (75 MHz, DMSO- $\left.d_{6}\right), \delta$ (ppm) 197.9, 181.4, 169.0, 168.7, 167.4, 143.3, 142.6, 140.6, 137.6, 133.6, 133.3, 133.1, 131.9, 129.7, 129.1, 128.9,
128.8, 128.5, 118.1, 117.4, 110.1, 60.6, 48.0, 44.8.; ESIMS: $m / z$ calculated for $\mathrm{C}_{29} \mathrm{H}_{21} \mathrm{~N}_{7} \mathrm{O}_{6} \mathrm{~S}$ $(\mathrm{M}+\mathrm{H})^{+} 596.13$ found 596.11, Anal. Calc. for $\mathrm{C}_{29} \mathrm{H}_{21} \mathrm{~N}_{7} \mathrm{O}_{6} \mathrm{~S}$ : C, 58.48; $\mathrm{H}, 3.55 ; \mathrm{N}, 16.46 \%$; found: C, 58.47; H, 3.53; N, 16.45\%.

1-(2-(5-((5-benzoyl-1H-benzo[d][1,2,3]triazol1-yl)methyl)-2-thioxo-1,3,4-oxadiazol-3(2H)yl)acetamido)-5-oxo-2-(p-tolyl)-2,5-dihydro1 H-pyrrole-3-carboxylic acid (6b)

Yield: $67 \%$; white solid, m.p. $182-183{ }^{\circ} \mathrm{C}$; ${ }^{1} \mathrm{H}$ NMR (300 MHz, DMSO- $d_{6}$ ), $\delta$ (ppm) 10.30 (s, 1H), $8.46-8.39(\mathrm{~m}, 1 \mathrm{H}), 8.14-8.05(\mathrm{~m}$, $1 \mathrm{H}), 7.89$ (dd, $J=10.2,2.3 \mathrm{~Hz}, 1 \mathrm{H}$ ), $7.86-7.76$ (m, 2H), $7.54-7.45(\mathrm{~m}, 1 \mathrm{H}), 7.22$ (dd, $J=7.9$, $0.9 \mathrm{~Hz}, 2 \mathrm{H}$ ), 6.98 (dd, $J=8.0,0.6 \mathrm{~Hz}, 2 \mathrm{H}), 6.69$ (d, $J=1.7 \mathrm{~Hz}, 1 \mathrm{H}), 5.56(\mathrm{~d}, J=1.6 \mathrm{~Hz}, 1 \mathrm{H}), 4.69$ 
(d, $J=17.6 \mathrm{~Hz}, 1 \mathrm{H}), 4.53-4.39(\mathrm{~m}, 2 \mathrm{H}), 4.23$ (d, $J=13.6 \mathrm{~Hz}, 1 \mathrm{H}), 2.30$ (s, 3H). ${ }^{13} \mathrm{C}$ NMR $(75$ MHz, DMSO- $d_{6}$ ), $\delta$ (ppm) 197.9, 181.4, 169.0, 168.7, 167.4, 143.3, 142.6, 140.6, 137.6, 136.8, 134.3, 133.6, 133.3, 131.9, 129.7, 129.5, 128.9, 128.5, 126.9, 118.1, 117.4, 110.1, 60.6, 48.1, 44.8, 18.9.; ESIMS: $\mathrm{m} / \mathrm{z}$ calculated for $\mathrm{C}_{30} \mathrm{H}_{23} \mathrm{~N}_{7} \mathrm{O}_{6} \mathrm{~S}(\mathrm{M}+\mathrm{H})+611.14$ found 611.12 Anal. Calc. $\mathrm{C}_{30} \mathrm{H}_{23} \mathrm{~N}_{7} \mathrm{O}_{6} \mathrm{~S}: \mathrm{C}, 59.11 ; \mathrm{H}, 3.80 ; \mathrm{N}$, 16.04\%; found: C, 59.09; H, 3.80; N, 16.03\%

1-(2-(5-((5-benzoyl-1H-benzo[d][1,2,3]triazol1-yl)methyl)-2-thioxo-1,3,4-oxadiazol-3(2H)yl)acetamido)-2-(4-methoxyphenyl)-5-oxo-2,5dihydro-1H-pyrrole-3-carboxylic acid (6c)

Yield: 54.7\%; white solid, m.p. $181-182$ ${ }^{\circ} \mathrm{C}$; ${ }^{1} \mathrm{H}$ NMR $\left(300 \mathrm{MHz}, \mathrm{DMSO}-d_{6}\right), \delta(\mathrm{ppm})$ 10.30 (s, 1H), $8.45-8.39$ (m, 1H), $8.14-8.04$ (m, $1 \mathrm{H}), 7.89$ (dd, $J=10.2,2.3 \mathrm{~Hz}, 1 \mathrm{H}), 7.86-$ $7.76(\mathrm{~m}, 2 \mathrm{H}), 7.65-7.53(\mathrm{~m}, 1 \mathrm{H}), 7.53-7.44$ (m, 2H), $7.31-7.21(\mathrm{~m}, 2 \mathrm{H}), 6.82-6.72(\mathrm{~m}$, $2 \mathrm{H}), 6.69(\mathrm{~d}, J=1.8 \mathrm{~Hz}, 1 \mathrm{H}), 5.60-5.53(\mathrm{~m}$, 1H), 4.69 (d, $J=17.6 \mathrm{~Hz}, 1 \mathrm{H}), 4.52-4.39$ (m, $2 \mathrm{H}), 4.23$ (d, $J=13.6 \mathrm{~Hz}, 1 \mathrm{H}), 3.81(\mathrm{~s}, 3 \mathrm{H}) .{ }^{13} \mathrm{C}$ NMR (75 MHz, DMSO- $\left.d_{6}\right), \delta$ (ppm) 197.9, 181.4, 169.0, 168.7, 167.4, 158.3, 143.3, 142.6, 140.6, 137.6, 133.6, 133.3, 131.9, 130.6, 129.7, 128.9, 128.5, 125.0, 118.1, 117.4, 114.2, 110.1, 60.6, 55.4, 48.1, 44.8.; ESIMS: $m / z$ calculated for $\mathrm{C}_{30} \mathrm{H}_{23} \mathrm{~N}_{7} \mathrm{O}_{7} \mathrm{~S}(\mathrm{M}+\mathrm{H})+627.14$ found 627.12 Anal. Calc. for $\mathrm{C}_{30} \mathrm{H}_{23} \mathrm{~N}_{7} \mathrm{O}_{7} \mathrm{~S}$ : C, 57.60; H, 3.71; $\mathrm{N}, 15.67 \%$; found: C, 57.59; $\mathrm{H}, 3.71 ; \mathrm{N}, 15.66 \%$.

1-(2-(5-((5-benzoyl-1H-benzo[d][1,2,3]triazol1-yl)methyl)-2-thioxo-1,3,4-oxadiazol-3(2H)yl)acetamido)-2-(4-chlorophenyl)-5-oxo-2,5dihydro-1H-pyrrole-3-carboxylic acid (6d)

Yield: 57.4\%; white solid, m.p. $176-177$ ${ }^{\circ} \mathrm{C}$; ${ }^{1} \mathrm{H}$ NMR (300 MHz, DMSO- $\left.d_{6}\right), \delta$ (ppm) 10.30 (s, 1H), $8.45-8.39$ (m, 1H), $8.14-8.04$ (m, 1H), 7.89 (dd, $J=10.2,2.3 \mathrm{~Hz}, 1 \mathrm{H}), 7.86-$ $7.76(\mathrm{~m}, 2 \mathrm{H}), 7.65-7.53(\mathrm{~m}, 1 \mathrm{H}), 7.53-7.44$ (m, 2H), $7.23-7.13(\mathrm{~m}, 2 \mathrm{H}), 7.13-7.06(\mathrm{~m}$, $2 \mathrm{H}), 6.69(\mathrm{~d}, J=1.8 \mathrm{~Hz}, 1 \mathrm{H}), 5.60-5.52(\mathrm{~m}$, $1 \mathrm{H}), 4.69(\mathrm{~d}, J=17.6 \mathrm{~Hz}, 1 \mathrm{H}), 4.52-4.39$ (m, $2 \mathrm{H}), 4.23(\mathrm{~d}, J=13.6 \mathrm{~Hz}, 1 \mathrm{H}) .{ }^{13} \mathrm{C}$ NMR $(75$
MHz, DMSO- $d_{6}$ ), $\delta$ (ppm) 197.9, 181.4, 169.0, 168.7, 167.4, 143.3, 142.6, 140.6, 137.6, 133.6, 133.3, 133.3, 132.1, 131.9, 129.7, 129.3, 128.9, 128.5, 127.8, 118.1, 117.4, 110.1, 60.6, 48.1, 44.8.; ESIMS: $m / z$ calculated for $\mathrm{C}_{29} \mathrm{H}_{22} \mathrm{ClN}_{7} \mathrm{O}_{6} \mathrm{~S}_{2}(\mathrm{M}+\mathrm{H})+630.09$ found 630.07 Anal. Calc. for $\mathrm{C}_{29} \mathrm{H}_{22} \mathrm{ClN}_{7} \mathrm{O}_{6} \mathrm{~S}_{2}: \mathrm{C}, 55.29 ; \mathrm{H}$, 3.20 ; N, 15.56\%; found: C, 55.29; H, 3.19; N, $15.54 \%$.

1-(2-(5-((5-benzoyl-1H-benzo[d][1,2,3]triazol1-yl)methyl)-2-thioxo-1,3,4-oxadiazol-3(2H)yl)acetamido)-5-oxo-2-(thiophen-2-yl)-2,5dihydro-1H-pyrrole-3-carboxylic acid (6e)

Yield: 56.1\%; white solid, m.p. $180-181$ ${ }^{\circ} \mathrm{C}$; ${ }^{1} \mathrm{H}$ NMR (300 MHz, DMSO- $\left.d_{6}\right), \delta(\mathrm{ppm})$ 10.30 (s, 1H), $8.45-8.39$ (m, 1H), $8.14-8.04$ (m, 1H), 7.89 (dd, $J=10.2,2.3 \mathrm{~Hz}, 1 \mathrm{H}), 7.86-$ 7.76 (m, 2H), $7.65-7.53$ (m, 1H), $7.53-7.44$ (m, 2H), $7.34(\mathrm{dd}, J=5.3,1.9 \mathrm{~Hz}, 1 \mathrm{H}), 6.98-$ $6.84(\mathrm{~m}, 3 \mathrm{H}), 5.56(\mathrm{dd}, J=1.7,0.5 \mathrm{~Hz}, 1 \mathrm{H}), 4.69$ (d, $J=17.6 \mathrm{~Hz}, 1 \mathrm{H}), 4.52-4.39(\mathrm{~m}, 2 \mathrm{H}), 4.23$ (d, $J=13.6 \mathrm{~Hz}, 1 \mathrm{H}) .{ }^{13} \mathrm{C}$ NMR $(75 \mathrm{MHz}$, DMSO$\left.d_{6}\right), \delta(\mathrm{ppm}) 197.9,181.4,172.2,168.7,165.6$, 143.3, 142.6, 137.9, 137.6, 135.3, 133.6, 133.3, 131.9, 131.3, 129.7, 128.9, 128.5, 126.2, 125.7, 124.8, 118.1, 110.1, 66.6, 48.1, 44.8.; ESIMS: $m / z$ calculated for $\mathrm{C}_{27} \mathrm{H}_{19} \mathrm{~N}_{7} \mathrm{O}_{6} \mathrm{~S}_{2}(\mathrm{M}+\mathrm{H}){ }^{+}$ 602.08 found 602.06 Anal. Calc. for $\mathrm{C}_{27} \mathrm{H}_{19} \mathrm{~N}_{7} \mathrm{O}_{6} \mathrm{~S}_{2}$ : C, 53.90; $\mathrm{H}, 3.18 ; \mathrm{N}, 16.30 \%$; found: C, 53.89; H, 3.17; N, 16.28\%.

1-(2-(5-)((5-benzoyl-1H-benzo[d][1,2,3]triazol1-yl)methyl)-2-thioxo-1,3,4-oxadiazol-3(2H)yl)acetamido)-2-(2-chlorophenyl)-5-oxo-2,5dihydro-1H-pyrrole-3-carboxylic acid (6f)

Yield: $64.8 \%$; white solid, m.p. 197 - 198 ${ }^{\circ} \mathrm{C}$; ${ }^{1} \mathrm{H}$ NMR (300 MHz, DMSO- $\left.d_{6}\right), \delta$ (ppm) 10.30 (s, 1H), $8.45-8.39$ (m, 1H), $8.14-8.05$ (m, 1H), $7.93-7.78(\mathrm{~m}, 3 \mathrm{H}), 7.65-7.55(\mathrm{~m}$, $2 \mathrm{H}), 7.54-7.46(\mathrm{~m}, 2 \mathrm{H}), 7.28-7.13(\mathrm{~m}, 3 \mathrm{H})$, $6.69(\mathrm{~d}, J=1.8 \mathrm{~Hz}, 1 \mathrm{H}), 5.56$ (dd, $J=1.8,0.6 \mathrm{~Hz}$, 1H), 4.69 (d, $J=17.6 \mathrm{~Hz}, 1 \mathrm{H}), 4.53-4.40$ (m, $2 \mathrm{H}), 4.23(\mathrm{~d}, J=13.6 \mathrm{~Hz}, 1 \mathrm{H}) .{ }^{13} \mathrm{C}$ NMR $(75 \mathrm{MHz}$, DMSO- $\left.d_{6}\right), \delta$ (ppm) 197.9, 181.4, 169.0, 168.7, 167.4, 143.3, 142.6, 140.9, 137.6, 134.2, 133.6, 
133.3, 132.9, 131.9, 131.8, 131.2, 130.1, 129.7, 128.9, 128.5, 127.8, 118.1, 117.8, 110.1, 59.5, 48.1, 44.8.; ESIMS: $\mathrm{m} / \mathrm{z}$ calculated for $\mathrm{C}_{29} \mathrm{H}_{20} \mathrm{ClN}_{7} \mathrm{O}_{6} \mathrm{~S}(\mathrm{M}+\mathrm{H})+630.09$ found 630.07 Anal. Calc. for $\mathrm{C}_{29} \mathrm{H}_{20} \mathrm{ClN}_{7} \mathrm{O}_{6} \mathrm{~S}$ : C, 55.29; H, 3.20; $\mathrm{N}, 15.56 \%$; found: C, 55.29; H, 3.19; N, 15.55\%.

1-(2-(5-((5-benzoyl-1H-benzo[d][1,2,3]triazol1-yl)methyl)-2-thioxo-1,3,4-oxadiazol-3(2H)yl)acetamido)-2-(4-ethylphenyl)-5-oxo-2,5dihydro-1H-pyrrole-3-carboxylic acid (6g)

Yield: 68.4\%; white solid, m.p. $204-206$ ${ }^{\circ} \mathrm{C}$; ${ }^{1} \mathrm{H}$ NMR $\left(300 \mathrm{MHz}\right.$, DMSO- $\left.d_{6}\right), \delta$ (ppm) 10.30 (s, 1H), $8.45-8.39$ (m, 1H), $8.13-8.06$ (m, 1H), $7.93-7.77(\mathrm{~m}, 3 \mathrm{H}), 7.64-7.45(\mathrm{~m}$, $3 \mathrm{H}), 7.14(\mathrm{dt}, J=8.2,1.0 \mathrm{~Hz}, 2 \mathrm{H}), 7.07-6.99$ $(\mathrm{m}, 2 \mathrm{H}), 6.69(\mathrm{~d}, J=1.8 \mathrm{~Hz}, 1 \mathrm{H}), 5.59-5.54(\mathrm{~m}$, $1 \mathrm{H}$ ), 4.69 (d, $J=17.6 \mathrm{~Hz}, 1 \mathrm{H}$ ), $4.52-4.40$ (m, $2 \mathrm{H}$ ), $4.23(\mathrm{~d}, J=13.6 \mathrm{~Hz}, 1 \mathrm{H}$ ), 2.74 (dddd, $J=$ 12.3, 6.1, 5.1, $4.1 \mathrm{~Hz}, 1 \mathrm{H}$ ), 2.50 (dqt, $J=12.3$, $5.1,1.0 \mathrm{~Hz}, 1 \mathrm{H}), 1.21(\mathrm{t}, J=5.1 \mathrm{~Hz}, 3 \mathrm{H}) \cdot{ }^{13} \mathrm{C}$ NMR (75 MHz, DMSO- $\left.d_{6}\right), \delta(\mathrm{ppm}) 197.9,181.4$, 169.0, 168.7, 167.4, 143.5, 143.3, 142.6, 140.6, 137.6, 134.7, 133.6, 133.3, 131.9, 129.7, 128.9, 128.9, 128.5, 126.8, 118.1, 117.4, 110.1, 60.6, 48.1, 44.8, 28.4, 15.3.; ESIMS: $m / z$ calculated for $\mathrm{C}_{31} \mathrm{H}_{25} \mathrm{~N}_{7} \mathrm{O}_{6} \mathrm{~S}(\mathrm{M}+\mathrm{H})+624.16$ found 624.16 Anal. Calc. for $\mathrm{C}_{31} \mathrm{H}_{25} \mathrm{~N}_{7} \mathrm{O}_{6} \mathrm{~S}$ : C, 59.70; $\mathrm{H}, 4.04$; $\mathrm{N}, 15.72 \%$; found: $\mathrm{C}, 59.69 ; \mathrm{H}, 4.04 ; \mathrm{N}, 15.71 \%$.

1-(2-(5-((5-benzoyl-1H-benzo[d][1,2,3]triazol1-yl)methyl)-2-thioxo-1,3,4-oxadiazol-3(2H)yl)acetamido)-2-(4-ethoxyphenyl)-5-oxo-2,5dihydro-1H-pyrrole-3-carboxylic acid (6h)

Yield: 64.7\%; white solid, m.p. $198-200$ ${ }^{\circ} \mathrm{C} ;{ }^{1} \mathrm{H}$ NMR (300 MHz, DMSO- $\left.d_{6}\right), \delta$ (ppm) 10.30 (s, 1H), $8.45-8.40$ (m, 1H), $8.14-8.03$ (m, 1H), $7.94-7.79(\mathrm{~m}, 3 \mathrm{H}), 7.66-7.45(\mathrm{~m}$, $2 \mathrm{H}), 7.30-7.22(\mathrm{~m}, 2 \mathrm{H}), 6.89-6.81(\mathrm{~m}, 2 \mathrm{H})$, $6.69(\mathrm{~d}, J=1.8 \mathrm{~Hz}, 1 \mathrm{H}), 5.56(\mathrm{~d}, J=1.5 \mathrm{~Hz}, 1 \mathrm{H})$, $4.69(\mathrm{~d}, J=17.6 \mathrm{~Hz}, 1 \mathrm{H}), 4.53-4.39(\mathrm{~m}, 2 \mathrm{H})$, $4.23(\mathrm{~d}, J=13.6 \mathrm{~Hz}, 1 \mathrm{H}), 4.12-3.92(\mathrm{~m}, 2 \mathrm{H})$, $1.33(\mathrm{t}, J=4.6 \mathrm{~Hz}, 3 \mathrm{H}) \cdot{ }^{13} \mathrm{C}$ NMR $(75 \mathrm{MHz}$, DMSO- $\left.d_{6}\right), \delta$ (ppm) 197.9, 181.4, 169.0, 168.7, 167.4, 158.6, 143.3, 142.6, 140.6, 137.6, 133.6, $133.3,131.9,130.8,129.7,128.9,128.5,128.2$,
118.0, 117.4, 115.4, 110.1, 63.5, 60.6, 48.1, 44.8, 14.8.; ESIMS: $\mathrm{m} / \mathrm{z}$ calculated for $\mathrm{C}_{31} \mathrm{H}_{25} \mathrm{~N}_{7} \mathrm{O}_{7} \mathrm{~S}(\mathrm{M}+\mathrm{H})+640.15$ found 640.13 Anal. Calc. for $\mathrm{C}_{31} \mathrm{H}_{25} \mathrm{~N}_{7} \mathrm{O}_{7} \mathrm{~S}$ : C, 58.21; H, 3.94; N, 15.33\%; found: C, 58.20; H, 3.93; N, 15.33\%.

\section{Result and discussion}

\section{Biology}

In vitro antimicrobial activity

$1 \mathrm{mg}$ of each molecule was dissolved in 1 $\mathrm{mL}$ of DMSO then made up to $10 \mathrm{~mL}$ with sterile water to obtain a concentration of 100 $\mu \mathrm{g} / \mathrm{mL}$. The microorganisms were maintained on nutrient agar media. The agar media was incubated with the different tested bacteria. After 24 to $48 \mathrm{~h}$ of incubation at $\sim 37 \stackrel{\circ}{\circ} \mathrm{C}$, dimethyl sulfoxide showed no inhibition zones. The diameters of the inhibition zones of the tested compounds were measured. This method was utilized to prove the minimum inhibitory concentration (MIC). The outcomes are outlined in Table 1.

The Synthesized compounds under study were screened for their antimicrobial activity using the cup plate method [37]. The bacteria screened were Staphylococcus, Bacillus, Escherichia coli and Pseudomonas aeruginosa and the fungi screened were Aspergillus niger and Candida albicans. Ciprofloxacin and clotrimazole were used as standards for antibacterial studies and antifungal studies respectively. The details are given in Table 1 . To analyze the impact of the nature of (R-), substitution on the antimicrobial activity, derivatives incorporating phenyl, $p$-chloro phenyl, $p$-methyl phenyl, $p$-methoxy phenyl, $o$ chlorophenyl, $p$-ethyl phenyl, $p$-ethoxy phenyl and 2-thienyl were synthesized. The synthesized compounds containing chloro and 2-thienyl group revealed excellent antimicrobial activity whereas the rest of the series showed good to moderate antimicrobial activity against the bacterial and fungal strain compared to standards. 


\section{Antibacterial and antifungal activity results}

The synthesized compounds were screened for their antimicrobial activity using the cup plate method [38]. The bacteria screened were Staphylococcus, Bacillus, Escherichia coli and Pseudomonas aeruginosa and the fungi screened were Aspergillus niger and Candida albicans. Ciprofloxacin and clotrimazole were used as standard for the antibacterial and antifungal studies, respectively. The results are presented in Table 1. To analyze the effect of the nature of
(Ar-) Substitution on the antimicrobial activity, derivatives incorporating phenyl, $p$ chloro phenyl, $p$-methyl phenyl, $p$-methoxy phenyl, $o$-chlorophenyl, $p$-ethyl phenyl, $p$ ethoxy phenyl and 2-thienyl were synthesized. The synthesized compounds containing chloro and 2-thienyl group revealed excellent antimicrobial activity whereas the rest of the series showed good to moderate antimicrobial activity against bacterial and fungal strain compared to standards (Figures 4 and 5).

Table 1. Results of in vitro antimicrobial activity

\begin{tabular}{ccccccc} 
Compound & \multicolumn{9}{c}{ Zone of inhibition (mm) } \\
& \multicolumn{7}{c}{ Bacteria screened } & \multicolumn{2}{c}{ Fungi screened } \\
& $\begin{array}{c}\text { Staphylococcus } \\
\text { aureus }\end{array}$ & $\begin{array}{c}\text { Bacillus } \\
\text { cereus }\end{array}$ & $\begin{array}{c}\text { Escherichia } \\
\text { coli }\end{array}$ & $\begin{array}{c}\text { Pseudomonas } \\
\text { aeruginosa }\end{array}$ & $\begin{array}{c}\text { Aspergillus } \\
\text { niger }\end{array}$ & $\begin{array}{c}\text { Candida } \\
\text { albicans }\end{array}$ \\
$\mathbf{6 a}$ & 15 & 16 & 13 & 16 & 17 & 13 \\
$\mathbf{6 b}$ & 24 & 16 & 24 & 17 & 19 & 13 \\
$\mathbf{6 c}$ & 16 & 17 & 18 & 15 & 16 & 15 \\
$\mathbf{6 d}$ & 17 & 18 & 13 & 15 & 16 & 17 \\
$\mathbf{6 e}$ & 22 & 15 & 22 & 20 & 25 & 27 \\
$\mathbf{6 f}$ & 24 & 17 & 23 & 11 & 17 & 14 \\
$\mathbf{6 g}$ & 12 & 10 & 15 & 14 & 16 & 19 \\
$\mathbf{6 h}$ & 13 & 11 & 16 & 11 & 18 & 20 \\
Ciprofloxacin & 25 & 23 & 27 & 22 & - & - \\
Clotrimazole & - & - & - & - & 26 & 28 \\
\hline
\end{tabular}

Boldfaced values indicate the active compounds; '-' indicates not tested

Figure 4. Antibacterial activity of synthesized compounds in comparison with the standard at the concentration of $100 \mu \mathrm{g} / \mathrm{mL}$

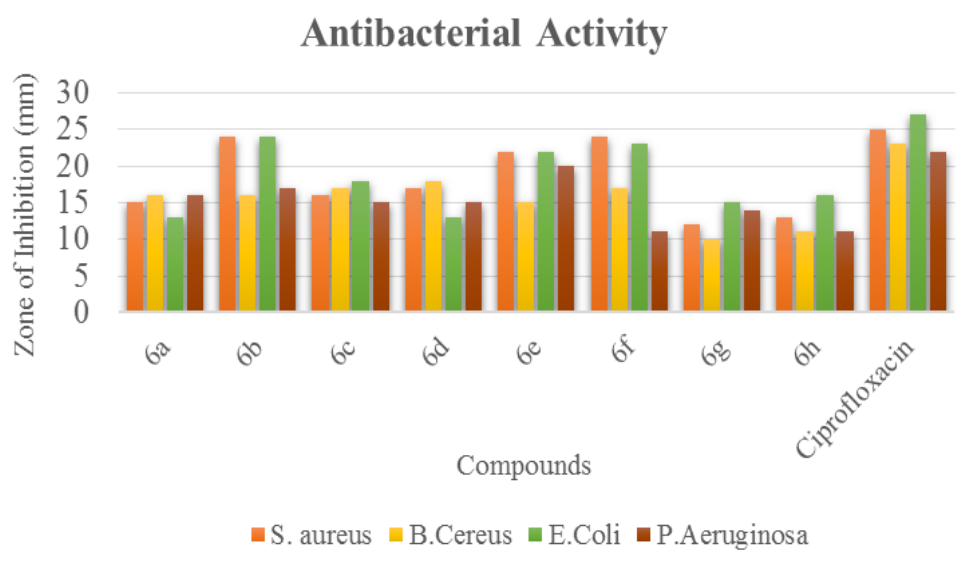


Figure 5. Antibacterial activity of synthesized compounds in comparison with the standard at the concentration of 100 $\mu \mathrm{g} / \mathrm{mL}$

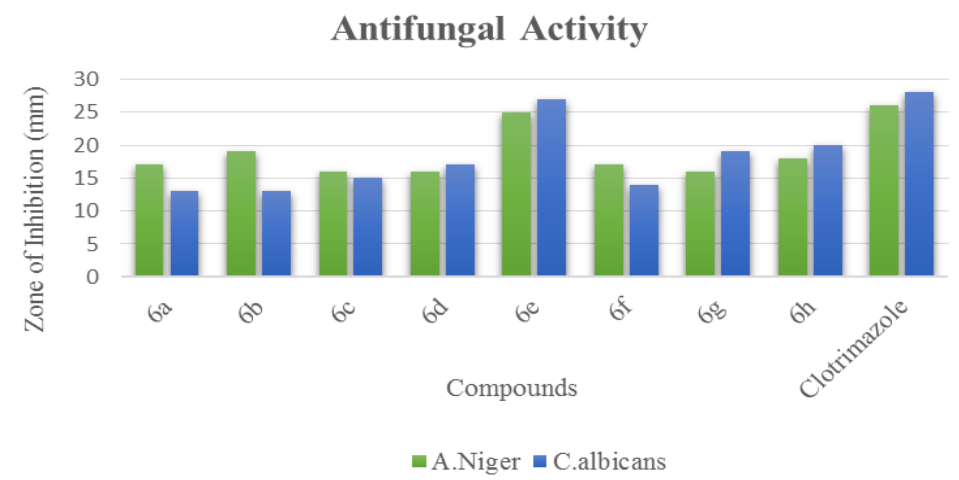

\section{In vitro antimycobacterial assay}

The antimycobacterial activity was assessed against the H37Rv employing microplate alamar blue assay procedure [41]. This method is nontoxic, using a thermally stable reagent. This method is explained as follows, $200 \mu \mathrm{L}$ of ultrapure water was added to whole outer perimeter wells of 96 sterile well plates to reduce drying up of the medium in the test wells while incubation. 96 plates taken $100 \mu \mathrm{L}$ of the Middlebrook 7H9 broth and consecutive dilution of the compounds was generated directly on the plate. The end drug concentrations confirmed were 100 to $0.190 \mu \mathrm{g} / \mathrm{mL}$. Plates were capped and sealed with parafilm and incubated at $37{ }^{\circ} \mathrm{C}$ for 5 days. $25 \mu \mathrm{L}$ of freshly made 1:1 mixture of alamar blue reagent and 10\% tween 85 was then added to the plate and incubated for about $24 \mathrm{~h}$. A blue color in the well was elucidated as no bacterial growth and a pink color was scored as growth. The potency is reported as MIC (minimum inhibitory concentration).

\section{Antimycobacterial activity result}

The developed molecules $\mathbf{6 a - h}$ were evaluated to in vitro antimycobacterial activity against MTB $H 37 \mathrm{v}$ employing the MABA method [40]. Ciprofloxacin and pyrazinamide were used as reference drugs. The outcomes of the in vitro antimycobacterial potency are outlined in Table 2 as MIC. Among all the synthesized molecules, $\mathbf{6 b}$ and $\mathbf{6 f}$ demonstrated a similar mycobacterial inhibitory potency at a MIC of $12.5 \mu \mathrm{M}$ compared to the standard drug ciprofloxacin (Figure 6).

\section{In silico ADME studies}

The aqueous solubility [39-42] of a molecule undoubtebly change its absorption and distribution tendency. Generally, a low solubility goes along with a poor absorption and therefore the common intention is to filter out less soluble molecules. Our predicted logs number was measured in mol/liter unit. From the literature it is evident that more than $85 \%$ of the drugs in the market have a (predicted) logS number greater than -4. As a essential merits of matter, lipophilicity is a characteristic used by scientists to estimate and interpret the transit and effect of chemicals in physiological systems. $\log P$ values are crucial to many companies and areas of research in evaluating how to transport chemical substances to particular sites. $\log P$ is employed in the pharmaceutical or biotech companies to explain the action of drug candidates in the body. Drug molecules are usually screened confering to $\log P$, some other benchmark also help to guide drug selection and optimization. 
Table 2. Antimycobacterial activity

\begin{tabular}{cc} 
Entry & $\begin{array}{c}\mathrm{a} I \mathrm{MIC}(\mu \mathrm{M}) \\
\mathrm{MTB}-\mathrm{H} 37 v\end{array}$ \\
$\mathbf{6 a}$ & 25.5 \\
$\mathbf{6 b}$ & 12.5 \\
$\mathbf{6 c}$ & 25.5 \\
$\mathbf{6 d}$ & 25.5 \\
$\mathbf{6 e}$ & 20.5 \\
$\mathbf{6 f}$ & 12.5 \\
$\mathbf{6 g}$ & 25.5 \\
$\mathbf{6 h}$ & 25.5 \\
Pyrazinamide & 3.15 \\
Ciprofloxacin & 12.5 \\
\hline
\end{tabular}

Figure 6. Graph Showing the

Antimycobacterial activity

antibacterial activity of synthesized compounds in comparison with the standard drugs

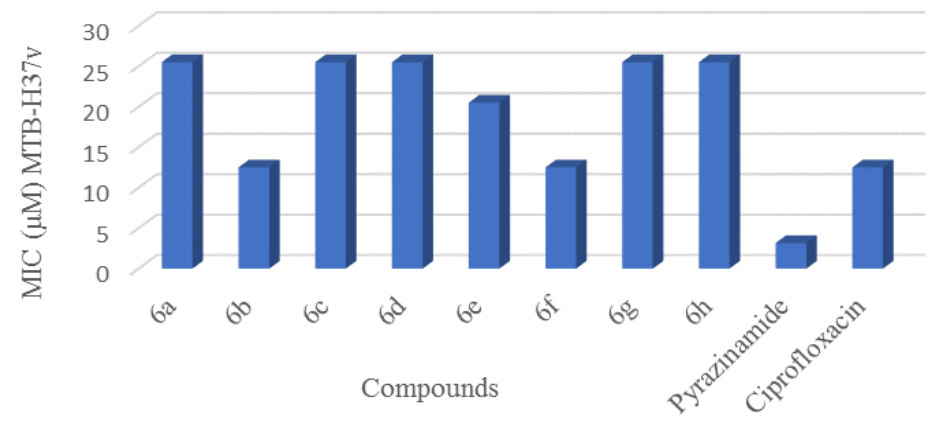

This is due to lipophilicity is a major determining key factor in a compound's absorption, distribution in the body, penetration across membranes and biological barriers, metabolism and excretion (ADME properties). A drug targeting CNS (central nervous system) should admirably have a $\log P$ value around 2 for oral and intestinal absorption the ideal value is 1.35-1.8, while a drug intended for sub-lingual absorption may have a $\log P$ value $>5$. $\log P$ help predict the likely transit of a molecule around the body, it also very useful in formulation, dosing, drug clearance, and toxicity. Though it is not the only deciding factor in these arguments, it plays a crucial role in helping scientists/researchers limit the liabilities of new drug molecules. There are many approaches around that assess a compound's drug likeness partially based on topological descriptors, fingerprints of MDL structure keys or other properties as $\operatorname{cLog} P$ and molecular weights. The distribution of drug likeness values calculated from https://www.organic-chemistry.org/prog/. Positive values of these synthesized compound showed to be a good drug candidates in the drug development process.

Lipinski's rule of five [42] also known as the Pfizer's rule of five or simply the rule of five (R05) is a rule of thumb to evaluate the druglikeness and determine if a chemical compound with a certain pharmacological or biological activity has properties that would make it a likely orally active drug in humans. The rule describes molecular properties important for a drug's pharmacokinetics in the human body, including their absorption, distribution, metabolism, and excretion ("ADME") components of the Lipinski's rule presented in Tables 3 and 4 . 
Table 3. Pharmacokinetic properties for good oral bioavailability for the compounds of $\mathbf{6 a}-\mathbf{h}$

\begin{tabular}{cccccccc} 
Entry & $\begin{array}{c}\text { Mol/PSA } \\
\mathrm{A}^{2}\end{array}$ & $\begin{array}{c}\mathrm{Mol} / \mathrm{Vol} \\
\mathrm{A}^{3}\end{array}$ & HBA & HBD & C/LogP & Mol/LogP & Mol/LogS \\
$\mathbf{6 a}$ & 133.69 & 573.99 & 10 & 2 & 0.35 & 2.67 & -7.24 \\
$\mathbf{6 b}$ & 133.69 & 591.18 & 10 & 2 & 0.96 & 3.39 & -8.20 \\
$\mathbf{6 c}$ & 133.69 & 594.93 & 10 & 2 & 0.7 & 3.07 & -7.70 \\
$\mathbf{6 d}$ & 141.24 & 605.83 & 11 & 2 & 0.28 & 2.76 & -7.50 \\
$\mathbf{6 e}$ & 134.25 & 586.00 & 11 & 2 & -0.44 & 2.50 & -7.04 \\
$\mathbf{6 f}$ & 133.69 & 590.97 & 10 & 2 & 0.96 & 3.27 & -7.94 \\
$\mathbf{6 g}$ & 133.69 & 613.07 & 10 & 2 & 1.11 & 3.57 & -8.27 \\
$\mathbf{6 h}$ & 140.82 & 624.40 & 11 & 2 & 0.69 & 3.24 & -7.94 \\
\hline
\end{tabular}

Table 4. Osiris calculation for bioavailability prediction of series 6a-h

\begin{tabular}{ccccc}
\multicolumn{5}{c}{ Bioavailability and drug score } \\
Entry & Solubility & Mol/Wt & Drug likeness & Drug score \\
$\mathbf{6 a}$ & -5.28 & 595.13 & 1.21 & 0.24 \\
$\mathbf{6 b}$ & -6.02 & 629.09 & 1.81 & 0.57 \\
$\mathbf{6 c}$ & -5.63 & 609.14 & 0.01 & 0.30 \\
$\mathbf{6 d}$ & -5.30 & 625.14 & 1.4 & 0.35 \\
$\mathbf{6 e}$ & -5.48 & 605.12 & 2.02 & 0.44 \\
$\mathbf{6 f}$ & -6.02 & 629.09 & 1.51 & 0.51 \\
$\mathbf{6 g}$ & -5.78 & 623.16 & 1.01 & 0.63 \\
$\mathbf{6 h}$ & -5.6 & 639.15 & 0.29 & 0.24 \\
\hline
\end{tabular}

Result of in silico ADME studies

These properties were calculated and discussed on the basis of Lipinski's rule [42] and its component. The compounds $\mathbf{6 a - h}$ fulfill Lipinski's rule and revealed good druglikeness score which is positive values (Table 4). $\mathrm{M} \log P$ of these compounds was found below 5 that means these shows good permeability across the cell membrane. The $\log S$ values are greater than -4 which predict that these molecules has good aqueous solubility. TPSA below $160 \AA$ Á2, $n$ violations $=1$ or $<0$ it means compound easily bind to the receptor, molecular mass $>500$, No. hydrogen bond donors $\leq 5$ (The sum of OHs and NHs), No. hydrogen bond acceptor $\leq 10$ (The sum of Os and Ns).

\section{Conclusion}

In this work, a series of novel heterocyclic compounds incorporating Pyrrole and benzotriazole-1,3,4-oxadiazole moieties were synthesized and characterized using ${ }^{1} \mathrm{H}$ NMR, ${ }^{13} \mathrm{C}$ NMR, mass spectroscopy and elemental analysis. The titled compounds were evaluated for their in vitro antimicrobial activity against six bacteria including two gram-positive, two gram-negative, and two fungal strains. All the compounds depicted good to moderate activity against the bacterial strain. Among all the compounds, $\mathbf{6 b}$ and $\mathbf{6 f}$ were found to be better antimycobacterial agents and $\mathbf{6 a}, \mathbf{6 b}$ and $\mathbf{6 e}$ were found to be excellent antifungal and antibacterial agent compared to rest of the series. The synthesized compounds also studied for their in-silico properties and showed good druglikeness properties.

\section{Statement of human and animal right}


This article does not contain any studies with human and animal subjects performed by any of the authors.

\section{Caution!}

Thiophosgene $\left(\mathrm{CSCl}_{2}\right)$ may cause severe dermatitis if allowed to come in contact with the skin. This preparation should be carried out in a good hood, and rubber gloves should be worn throughout.

Harmful if swallowed.

Causes skin irritation.

Causes serious eye irritation.

Toxic if inhaled.

May cause respiratory irritation.

Wear protective gloves/protective clothing/eye protection/face protection.

\section{Acknowledgement}

The authors would like to appreciate the Principal and Head Department of Chemistry of Arts, Science and Commerce College for providing research laboratory and library facilities. The authors also thank Prof. Dr. K. R. Desai for encouragement and guidance during the research work. The authors would like to thank Department of Chemistry, M.B.Patel Science College and Department of Organic Chemistry, A.N. Patel P. G. Institute, Anand.

\section{Disclosure statement}

No potential conflict of interest was reported by the authors.

\section{ORCID}

Keyur M. Pandya (D: 0000-0002-1282-7078

\section{References}

[1] M.L. Barreca, A. Rao, L. De Luca, M. Zappala, C. Gurnari, P. Monforte, E. De Clercq, B.V. Maele, Z. Debyser, M. Witvrouw, J.M. Briggs, A. Chimirri, J. Chem. Inform. Comput. Sci., 2004, 44, 1450-1455.
[2] K. Pandya, P.S. Desai, World J. Pharm. Res., 2018, 7, 465-474.

[3] N. Kolocouris, A. Kolocouris, G.B. Foscolos, G. Fytas, J. Neyts, E. Padalko, J. Balzarini, R. Snoeck, G. Andrei, E. De Clercq. J. Med. Chem., 1996, 39, 3307-3318.

[4] K.M. Pandya, P.S. Desai, N.B. Patel, B.P. Dave, Chem. Biol. Interface, 2018, 8, 314322

[5] V.O. Koz'minykh, N.M. Igidov, S.S. Zykova, V.E. Kolla, N.S. Shuklina, T. Odegova, Pharm. Chem. J., 2002, 36, 188-191

[6] K.M. Pandya, Synthesis and Cytotoxicity of Azaheterocyclic Compounds. Thesis, Rowan University: New Jersey, 2018.

[7] T.A. Silina, V.L. Gein, L.F. Gein, E.V. Voronina, Pharm. Chem. J., 2003, 37, 585587.

[8] V.L. Gein, E.V. Voronina, T.E. Ryumina, G.N. Novoselova, K.D. Potemkin, Y.S. Andreichikov, Pharm. Chem. J., 1996, 30, 25-26.

[9] F. Lovren, I.D. Gaon, B. Bobarevic, Arch. Pharm., 1990, 323, 901-904.

[10] T. Sano, Y. Horiguchi, J. Toda, K. Imafuku, Y. Tsuda, Chem. Pharm. Bull., 1984, 32, 497-503.

[11] A.J. Aasen, C.C. Culvenor, J. Org. Chem., 1969, 34, 4143- 4147.

[12] B.M. Goldschmidt, Substituted pyrrolizidines, J. Org. Chem., 1962, 27, 4057-4058.

[13] J.J. Tufariello, J.P. Tette, J. Org. Chem., 1975, 40, 3866-3869.

[14] K. M. Pandya, A.H. Patel, P.S.Desai, Chem Afr., 2019. https://doi.org/10.1007/s42250-01900096-5

[15] C.J.H. Morton, R. Gilmour, D.M. Smith, P. Lightfoot, A.M.Z. Slawin, E. MacLean, Tetrahedron, 2002, 58, 5547-5565.

[16] A. Ueda, Y. Sekiya, M. Taniguchi, Japanese Patent JP 2,003,165,918, 2003.

[17] K. Pandya, R. Patel, J. Chem. Chem. Sci., 2017, 7, 1331-1341. 
[18] J.M. Adam, P.V. Dalvi, V.S. Ekkundi, J.P. Bacher, S. Tiwari, World Patent WO 2,004,083,170, 2004.

[19] J.M. Adam, P.V. Dalvi, V.S. Ekkundi, J.P. Bacher, R. Sreenivasan, D.M. Rane, World Patent WO 2,004,089,941, 2004.

[20] H. Shiraishi, T. Nishitani, S. Sakaguchi, Y. Ishii, J. Org. Chem., 1998, 63, 6234-6238.

[21] X. Lin, Z. Mao, X. Dai, P. Lu, Y. Wang, Chem. Commun., 2011, 47, 6620-6622.

[22] O.A. Attanasi, G. Favi, F. Mantellini, G. Moscatelli, S. Santeusanio, J. Org. Chem. 2011, 76, 2860-2866.

[23] E. Ghabraie, S. Balalaie, M. Bararjanian, H.R. Bijanzadeh, F. Rominger, Tetrahedron, 2011, 67, 5415-5420.

[24] C.R. Reddy, M.D. Reddy, B. Srikanth, K.R. Prasad, Org. Biomol. Chem., 2011, 9, 60276033.

[25] B.M. Trost, J.-P. Lumb, J.M. Azzarelli, J. Am. Chem. Soc., 2011, 133, 740-743.

[26] A.V. Gulevich, A.S. Dudnik, N. Chernyak, V. Gevorgyan, Chem. Rev., 2013, 113, 30843213.

[27] H.C. Brown, U.R. Khire, G. Narla, U.S. Racherla, J. Org. Chem., 1995, 60, 544-549.

[28] O. Yuryeva, Y. Kondratova, L. Logoyda, Asian J. Pharm. Clin. Res., 2018, 11, 200204.

[29] A.R. Karimi, Z. Alimohammadia, J. Azizian, A.A. Mohammadi, M.R. MohammadiZadeh, Catal. Commun., 2006, 7, 728-732.
[30] A.O. Eseola, W. Li, W.H. Sun, M. Zhang, L. Xiao, J. A. Woods, Dyes Pigments, 2011, 88, 262-273.

[31] R. Hosseinnia, M. Mamaghani, K. Tabatabaeian, F. Shirini, M. Rassa, Bioorg. Med. Chem. Lett., 2012, 22, 5956-5960.

[32] B. Yilmaz, U. Kocak, J. Adv. Pharm. Res., 2019, 3, 17-22.

[33] D.A. Dougherty, Acc. Chem. Res., 2013, 46, 885-893.

[34] R. Leon, A.G. Garcia, J. Marco-Contelles, Med. Res. Rev., 2013, 33, 139.

[35] M. Metwally, M. Gouda, A.N. Harmal, A. Khalil, Eur. J. Med. Chem., 2012, 56, 254262.

[36] H.P. Shah, B.R. Shah, J.J. Bhatt, N.C. Desai, P.B. Trivedi, N.K. Undavia, Indian. J. Chem. B, 1998, 37, 180-182.

[37] S.M. El-Khawas, N.S. Habib, J. Hetero. Chem., 1989, 26, 177-181.

[38] R.R. Somani, P.Y. Shirodkar, V.J. Kadam, Chin. J. Chem., 2008, 26, 117-1731.

[39] D.K. Shukla, S.D. Srivastava, Indian J. Chem., 2008, 47, 463-469.

[40] M. Tabcheh, M. Baroudi, F. El-omar, A. Elzant, M. Elkhatib, V. Rolland, Asian J. Chem., 2006, 18, 1771-1782.

[41] S.B. Rose, R.B. Miller, J. Bacteriol., 1939, 38, 525-537.

[42] C.A. Lipinski, F. Lombardo, B.W. Dominy, P.J. Feeney, Adv. Drug Deliv. Rev., 2001, 46, 3-26.

How to cite this manuscript: Keyur Pandya, Bhavesh Dave, Rajesh Patel, Piyush Desai, In Silico Approach Towards the Prediction of Drug-likeness, in Vitro Microbial Investigation and Formation of Dihydropyrrolone Conjugates, Adv. J. Chem. A, 2020, 3(4), 378-390.

Copyright (C) 2020 by SPC (Sami Publishing Company)+ is an open access article distributed under the Creative Commons Attribution License, which permits unrestricted use, distribution, and reproduction in any medium, provided the original work is properly cited. 